\title{
TREM2/DAP12 Complex Regulates Inflammatory Responses in Microglia via the JNK Signaling Pathway
}

\author{
Li Zhong $^{1 \dagger}$, Zhen-Lian Zhang ${ }^{1 \dagger}$, Xinxiu Li ${ }^{1 \dagger}$, Chunyan Liao ${ }^{1 \dagger}$, Pengfei Mou ${ }^{1}$, \\ Tingting Wang ${ }^{1}$, Zongqi Wang ${ }^{1}$, Zhe Wang ${ }^{1}$, Min Wei ${ }^{1}$, Huaxi $\mathrm{Xu}^{1,2}$, Guojun $\mathrm{Bu}^{1,3 *}$ \\ and Xiao-Fen Chen ${ }^{1,4 *}$
}

${ }^{1}$ Fujian Provincial Key Laboratory of Neurodegenerative Disease and Aging Research, Institute of Neuroscience, Medical College, Xiamen University, Xiamen, China, ${ }^{2}$ Neuroscience and Aging Research Center, Sanford-Burnham-Prebys Medical Discovery Institute, La Jolla, CA, United States, ${ }^{3}$ Department of Neuroscience, Mayo Clinic, Jacksonville, FL, United States, ${ }^{4}$ Shenzhen Research Institute of Xiamen University, Shenzhen, China

OPEN ACCESS

Edited by:

Yu Tang,

University of Texas Southwestern Medical Center, United States

Reviewed by: Robert Vassar, Northwestern University, United States

Midori A. Yenari, University of California, San Francisco, United States

*Correspondence: Guojun Bu bu.guojun@mayo.edu Xiao-Fen Chen chenx@xmu.edu.cn

${ }^{+}$Co-first authors. These authors have contributed equally to this work.

Received: 07 March 2017 Accepted: 06 June 2017 Published: 21 June 2017

Citation: Zhong L, Zhang Z-L, Li X, Liao C, Mou P, Wang T, Wang Z, Wang $Z$, Wei $M, X u H, B u G$ and Chen X-F (2017) TREM2/DAP12 Complex Regulates Inflammatory Responses in Microglia via the JNK Signaling Pathway.

Front. Aging Neurosci. 9:204. doi: 10.3389/fnagi.2017.00204
DNAX-activating protein of $12 \mathrm{kDa}$ (DAP12) is a signaling adapter protein expressed in cells that participate in innate immune responses. By pairing with different triggering receptors expressed on myeloid cell (TREM) proteins, DAP12 can mediate both positive and negative cellular responses. In particular, TREM1 acts as an amplifier of the immune response, while TREM2 functions as a negative regulator. TREM2 has also been shown to stimulate the phagocytosis of apoptotic neurons and define the barrier function in microglia. Notably, loss-of-function mutations of either DAP12 or TREM2 result in a disorder known as Nasu-Hakola disease (NHD); and mutations of these genes have been associated with the risk for Alzheimer's disease (AD), suggesting that TREM2 and DAP12 may regulate common signaling pathways in the disease pathogenesis. In this study, we demonstrated an anti-inflammatory role of DAP12 in murine microglia that depends on the presence of TREM2. We also uncovered the JNK signaling pathway as the underlying molecular mechanism by which the TREM2/DAP12 complex suppresses the hyperactivation of microglia upon LPS stimulation. Interestingly, LPS down-regulates the expression of Trem2 via the activation of JNK and NF-kB signaling pathways, resulting in a vicious cycle that synergistically promotes the inflammatory responses. Our study provides insights into mechanism-based therapy for neuroinflammatory disorders.

Keywords: DAP12, TREM2, JNK, LPS, microglia, inflammation

\section{INTRODUCTION}

DNAX-activating protein of $12 \mathrm{kDa}$ (DAP12; also known as TYROBP and KARAP) is a signaling adapter protein expressed by a variety of innate immune cells including macrophages, microglia, monocytes, dendritic cells and natural killer (NK) cells (Lanier, 2009). The broad distribution of DAP12 suggests a general function in immune responses. DAP12 consists of a minimal extracellular domain, a transmembrane segment and a cytoplasmic region containing a single immunoreceptor tyrosine-based activation motif (ITAM). An aspartic acid in the transmembrane domain of DAP12 allows its association with cell surface receptors via an electrostatic interaction. The receptors usually have an oppositely charged amino acid (arginine or lysine) embedded within their transmembrane region that allows the formation of non-covalent complexes with DAP12 (Lanier and Bakker, 2000; Humphrey et al., 2005). Ligation of a DAP12-associated receptor to its ligand leads to the activation of SRC-family kinases and 
subsequent phosphorylation of tyrosine residues in the ITAM of DAP12 (Mason et al., 2006). DAP12 was originally shown to trigger the activation of NK cells (Lanier et al., 1998). Since then, more than 20 DAP12-associated receptors have been identified (Turnbull and Colonna, 2007). Triggering receptors expressed on myeloid cells (TREMs) are a family of cell surface receptors expressed broadly on myeloid cells that have been identified to associate with DAP12 (Bouchon et al., 2000; Daws et al., 2001; Chung et al., 2002). In particular, TREM1 is a potent amplifier of the inflammatory responses; while TREM2 has an anti-inflammatory function (Bouchon et al., 2000; Gibot et al., 2004; Takahashi et al., 2005; Turnbull et al., 2006).

TREM2 is a DAP12-coupled receptor that acts as a sensor for a wide array of lipids and apolipoprotein $\mathrm{E}$ (ApoE) in the central nervous system (CNS; Atagi et al., 2015; Bailey et al., 2015; Wang et al., 2015; Yeh et al., 2016). Notably, loss-of-function mutations of either DAP12 or TREM2 result in a disorder known as Nasu-Hakola disease (NHD; Paloneva et al., 2000, 2002). Furthermore, both TREM2 (Guerreiro et al., 2013; Jonsson et al., 2013) and DAP12 (Pottier et al., 2016) mutations have been found to be associated with the risk for Alzheimer's disease (AD). These observations suggest that TREM2 and DAP12 may regulate common signaling pathways in the disease pathogenesis. TREM2 and DAP12 are both preferentially expressed in microglia within the CNS (Sessa et al., 2004). Together, they regulate functions in microglia including inhibition of pro-inflammatory responses and stimulation of phagocytosis of apoptotic neurons
(Takahashi et al., 2005; Hamerman et al., 2006; Turnbull et al., 2006; Zhong et al., 2015). Recently, TREM2/DAP12 complex has also been demonstrated to regulate the barrier function in microglia that prevents the outward extension of amyloid fibrils and axonal dystrophy (Sirkis et al., 2016; Yuan et al., 2016).

Despite intense interest in the function of TREM2/DAP12 complex in microglia, current understanding of the relevant molecular, cellular and biophysical mechanisms is limited. Studies elucidating such mechanisms may uncover targetable pathways for $\mathrm{AD}$ therapy. In this study, we demonstrated an anti-inflammatory role of DAP12 in murine microglia that requires the function of TREM2. Mechanistically, TREM2/DAP12 suppressed the hyperactivation of JNK signaling pathway upon LPS stimulation. Consequently, a JNK inhibitor, SP600125, eliminated the hypersensitivity of Dap12-deficient microglia to LPS. Together, our data suggest that TREM2/DAP12 complex negatively regulates LPS-induced inflammatory responses by modulating the JNK signaling pathway in microglia.

\section{MATERIALS AND METHODS}

\section{Reagents and Antibodies}

Amaxa ${ }^{\circledR}$ Cell Line Nucleofector ${ }^{\circledR}$ Kit $\mathrm{T}$ and Amaxa ${ }^{\circledR}$ Glia Cell Nucleofector ${ }^{\circledR}$ Kit $\mathrm{T}$ were purchased from LONZA. Primers for quantitative RT-PCR were synthesized by Life Technologies. SYBR Green for quantitative RT-PCR was

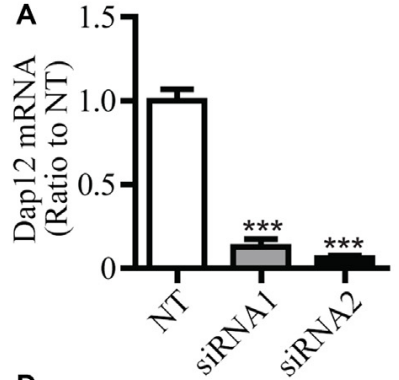

D

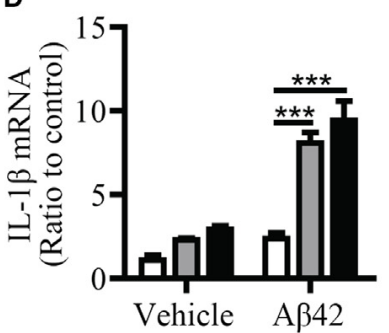

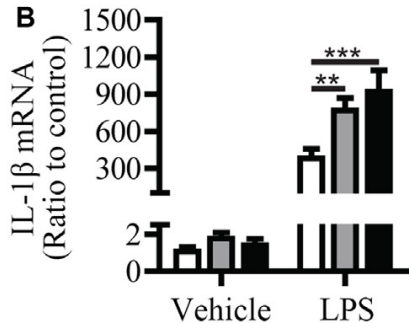

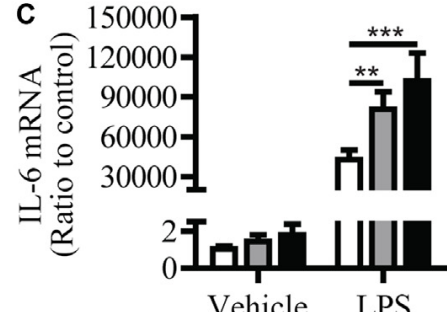

E

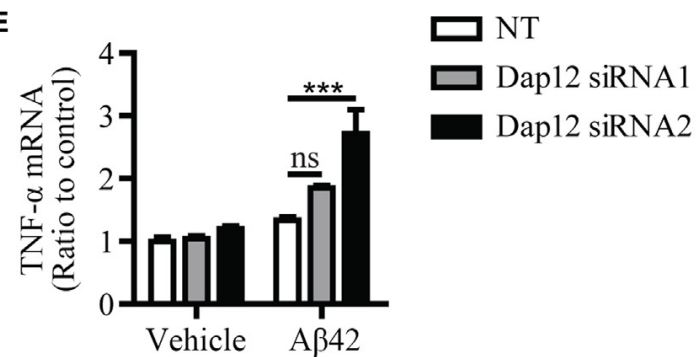

FIGURE 1 | Knockdown of Dap12 exacerbates LPS- or A 342 -oligomer-stimulated production of pro-inflammatory cytokines. (A) Primary microglia cells were transiently transfected with non-targeting siRNA (NT) or Dap12-specific siRNAs for $48 \mathrm{~h}$. The relative mRNA levels of Dap12 were determined by quantitative RT-PCR and shown as bar graph ( $n=3$, 1-way ANOVA). (B,C) Cells from (A) were treated with $500 \mathrm{ng} / \mathrm{mL}$ LPS or vehicle control for 4 h. RNA was extracted and the relative mRNA levels of IL-1 $\beta$ (B) and IL-6 (C) shown as bar graph were determined by quantitative RT-PCR ( $n=3$, two-way ANOVA). (D-E) Cells from (A) were treated with $10 \mu \mathrm{M}$ oligomeric-A $\beta 42$ or vehicle control for $4 \mathrm{~h}$. RNA was extracted and the relative mRNA levels of IL-1 $\beta$ and TNF- $\alpha$ shown as bar graph were determined by quantitative RT-PCR $\left(n=3\right.$, two-way ANOVA). $\beta$-actin was used as an internal control. Data represent mean \pm SEM. ${ }^{* *} p<0.01$; *** $p<0.001$; ns, not significant. 
purchased from Roche. SP600125, Bay11-7082, SB203580, U0126 and LPS were purchased from Sigma. Amyloid- $\beta 42$ (A $\beta 42)$ peptide was purchased from AnaSpec. Oligomeric A 342 was prepared as previously described (Huang et al., 2015). Antibodies used in this study are as followed: antiphospho-p38-MAPK, anti-total-p38-MAPK, anti-phosphoERK1/2, anti-total-ERK1/2, anti-phospho-JNK, anti-total-JNK,

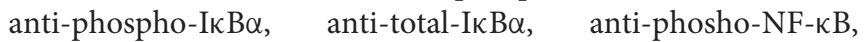
anti-total-NF- $\kappa \mathrm{B}$, anti-phospho-c-Jun, anti-total-c-Jun and anti- $\beta$-actin were purchased from Cell Signaling Technology; anti-tubulin (Millipore); anti-mouse IgG and anti-rabbit IgG antibody conjugated with horseradish peroxidase (ThermoFisher Scientific).

\section{Isolation and Culture of Mouse Primary Microglia}

Trem 2 knockout mice (Trem2-KO on C57BL/6N background) and wild-type (WT) C57BL/6N mice were obtained from the UC Davis Knockout Mouse Project (KOMP) repository. The exons 2-4 of the Trem2 gene were replaced with a $\mathrm{LacZ}$ reporter which is identical to the line recently reported
(Jay et al., 2015). Primary microglial cultures were prepared as previously described (Zhu et al., 2010; Atagi et al., 2015). All animal experiments were conducted in compliance with the protocols approved by the Institutional Animal Care and Use Committee of Xiamen University. Briefly, WT or Trem2-KO mice (3-4 pups) at postnatal day 1-2 were used to prepare mixed glial cultures. Cells were plated onto flasks and grown in DMEM supplemented with $10 \%$ heat-inactivated fetal bovine serum (FBS; Gibco). Three days later, medium was changed to that containing $25 \mathrm{ng} / \mathrm{mL}$ GM-CSF and $10 \%$ FBS. Primary microglia were harvested by shaking (200 rpm, $20 \mathrm{~min})$ after 10-12 days in culture and once every 3 days thereafter (up to three harvests).

\section{Western Blotting}

BV2 microglial cells or primary microglia were lysed at the indicated times with lysis buffer ( $1 \%$ NP-40, $50 \mathrm{mM}$ Tris$\mathrm{HCl}, \mathrm{pH} 8.0,150 \mathrm{mM}$ sodium chloride) supplemented with protease and phosphotase inhibitor cocktails. BCA protein assay kit was used to determine the protein concentration according to the manufacturer's instruction (ThermoFisher
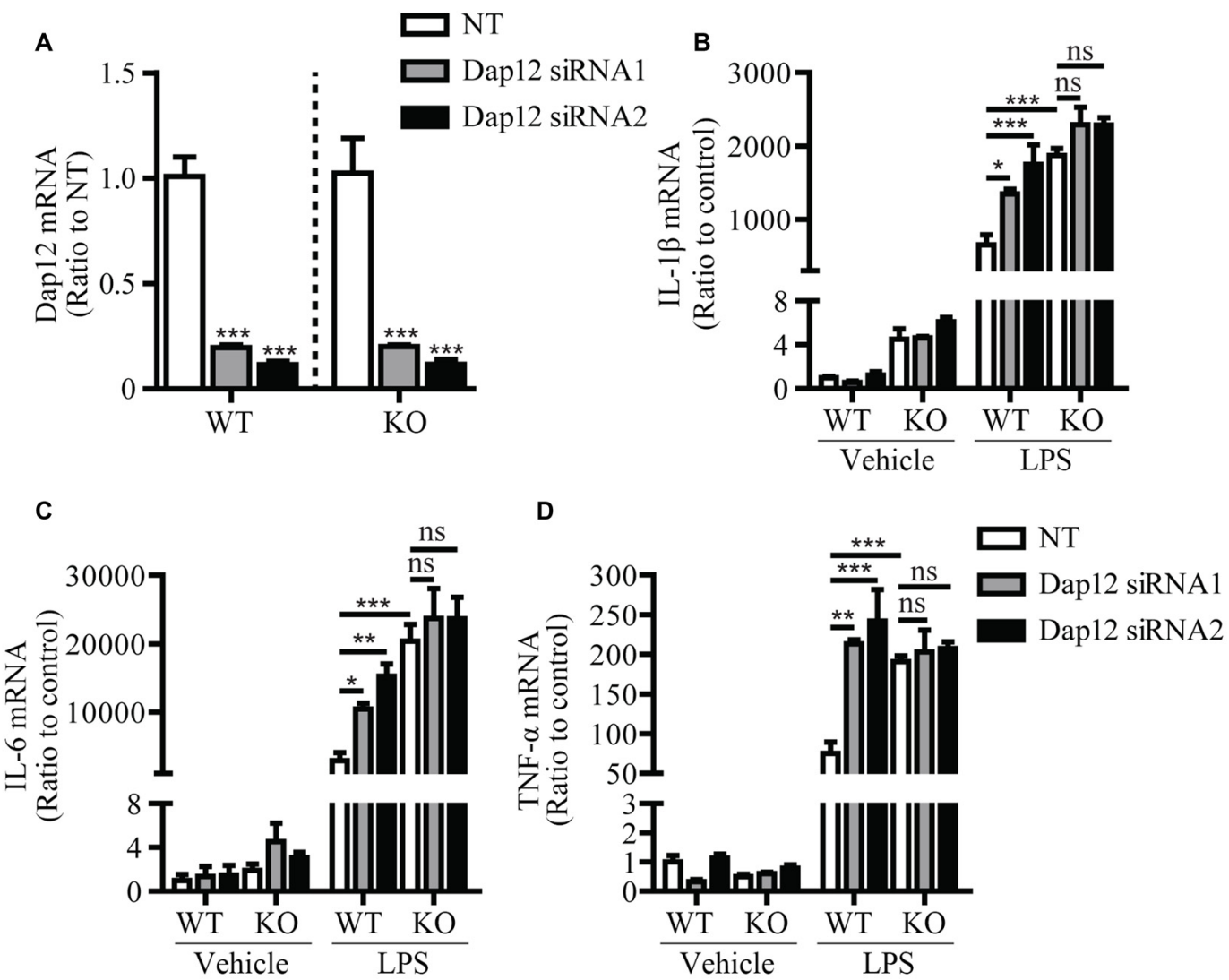

FIGURE 2 | DAP12 regulates inflammatory responses in a manner that depends on the presence of TREM2. (A) Primary microglia cells from wild-type (WT) or Trem2- knockout (KO) mice were transiently transfected with NT or Dap12-specific siRNAs for 48 h. The relative mRNA levels of Dap12 were determined by quantitative RT-PCR and shown as bar graph ( $n=3$, one-way ANOVA). (B-D) Cells from (A) were treated with $100 \mathrm{ng} / \mathrm{mL} L P S$ or vehicle control for $4 \mathrm{~h}$. RNA was extracted and the relative mRNA levels of IL-1 $\beta$ (B) IL-6 (C) TNF- $\alpha$ (D) shown as bar graph were determined by quantitative RT-PCR ( $n=3$, two-way ANOVA). $\beta$-actin was used as an internal control. Data represent mean \pm SEM. ${ }^{*} p<0.05 ;{ }^{* *} p<0.01 ;{ }^{* * *} p<0.001$; ns, not significant. 
Scientific). Equal amounts of total proteins were analyzed by sodium dodecyl sulfate-polyacrylamide gel electrophoresis (SDS-PAGE) and Western blotting using appropriate antibodies and HRP-conjugated secondary antibodies. Proteins were visualized using ECL Western blotting detection reagents (Millipore). Immunoreactive bands were quantified using ImageJ.

\section{Quantitative RT-PCR}

Total RNAs were extracted using TRIzol reagent (Invitrogen). One microgram RNA was reverse-transcribed into firststrand cDNA using TransScript All-in-One First-Strand cDNA Synthesis SuperMix (TRANSGEN BIOTECH, Beijing, China) according to the manufacturer's protocol. Quantitative PCR was performed using the FastStart Universal SYBR Green Master (Roche). The primer sequences used for Dap12, Trem2, IL-1 $\beta$, TNF- $\alpha$, IL- 6 and $\beta$-Actin were the same as previously described (Zhong et al., 2015, 2017).

\section{RNA Interference}

siRNA at a concentration of $300 \mathrm{nM}$ was transfected into BV2 cells using Amaxa ${ }^{\circledR}$ Cell Line Nucleofector ${ }^{\circledR}$ Kit $\mathrm{T}$ or primary microglia cells using Amaxa ${ }^{\circledR}$ Glia Cell Nucleofector ${ }^{\circledR}$ Kit T. Cells were harvested $48 \mathrm{~h}$ later, followed by RNA extraction for quantitative RT-PCR analysis or protein extraction for Western blotting analysis. The siRNA sequences for Dap12 were the same as previously described (Zhong et al., 2015).

\section{Statistical Analyses}

Statistical analyses were performed using GraphPad Prism and all data were presented as mean \pm SEM. At least three independent experiments were analyzed by unpaired $t$-test, one-way ANOVA or two-way ANOVA test. To classify and indicate significant values, the following $p$-values were used: ${ }^{*} p<0.05 ;{ }^{* *} p<0.01$; *** $p<0.001 ;$ ns, not significant.

\section{RESULTS}

\section{DAP2 Inhibits LPS-Induced Cytokines Production Dependent on TREM2 Receptor}

In our previous study, we found that knockdown of Dap12 gene in microglial BV2 cells significantly increased the mRNA levels of pro-inflammatory cytokines in the presence of LPS (Zhong et al., 2015). To further confirm the role of DAP12 in mediating the inflammatory responses to pathogenic stimuli, we employed two Dap12-specific siRNAs to knockdown the expression of Dap12 in primary microglia and examined its impacts on cytokine expression (Figure 1A). Consistently, the knockdown of Dap12 significantly increased the mRNA levels of IL-1 $\beta$ and IL- 6 in LPS-stimulated primary microglia (Figures 1B,C). The production of IL-1 $\beta$ and TNF- $\alpha$ were also elevated in response to treatment with A 442 oligomers in Dap12-knockdown primary microglia

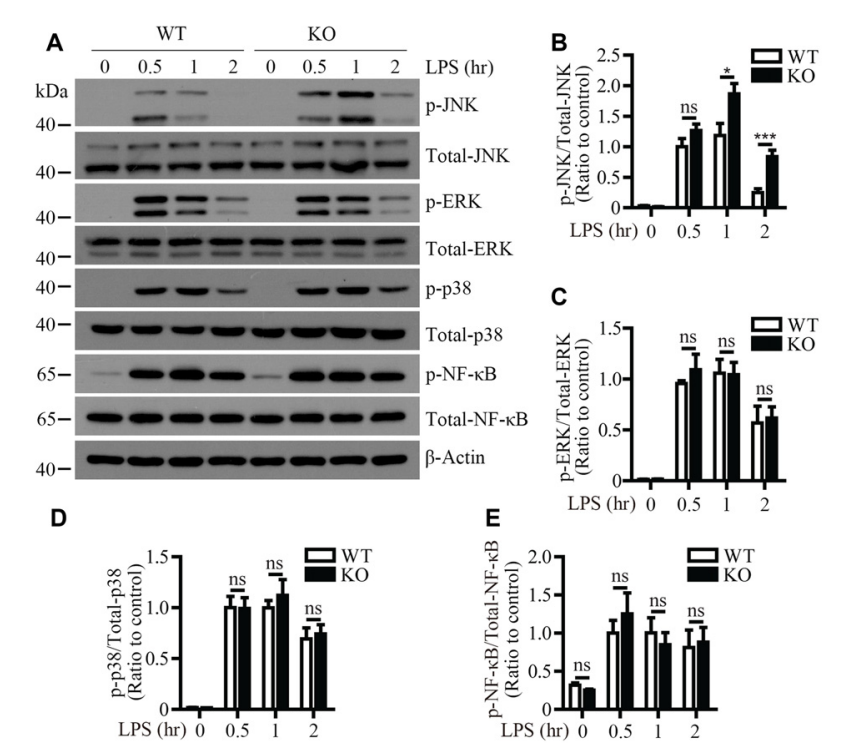

FIGURE 3 | LPS-induced JNK phosphorylation is increased in Trem2-deficient microglia. (A) Primary microglia cells from WT or Trem2- knockout (KO) mice were stimulated with $100 \mathrm{ng} / \mathrm{ml}$ LPS for the indicated time. Cell lysates at each time point were analyzed by Western blotting using antibodies specific for either total proteins or phosphorylated form of JNK, P38-MAPK, ERK1/2 and NF-KB. (B-E) Bar graphs show the quantification of Western blots as ratios of phospho-JNK/total JNK (B) phospho-ERK1/2/total ERK1/2 (C) phospho-p38-MAPK/total p38-MAPK (D) and phospho-NF-кB/total NF- $\mathrm{KB}(\mathbf{E})$, respectively. $\beta$-actin was used as an internal control. The ratio at " $0.5 \mathrm{~h}$ " time point of WT cells served as a control $(n \geq 3$, unpaired Student's $t$-test). Data represent mean \pm SEM. ${ }^{*} p<0.05$; ${ }^{* * *} p<0.001$; ns, not significant.

(Figures 1D,E). These data suggest that DAP12 is essential for suppressing the production of pro-inflammatory cytokines when microglial cells are exposed to pathogenic stimuli.

In cells of myeloid origin, TREM1 and TREM2 are two receptors that signal through DAP12 to oppositely regulate the inflammatory response. TREM1 has been shown to function as an amplifier of the inflammatory response (Bouchon et al., 2000), whereas TREM2 has an anti-inflammatory function (Turnbull et al., 2006). Since we observed an anti-inflammation function of DAP12 in microglia, we further investigated whether DAP12 suppresses the production of inflammatory cytokines in a manner that depends on TREM2. Primary microglia were isolated from both WT and Trem2-knockout (KO) mice and further subjected to siRNA treatment that specifically knock down the expression of Dap12 (Figure 2A). Although the deficiency of Dap12 significantly enhanced the production of inflammatory cytokines IL-1 $\beta$, IL- 6 and TNF- $\alpha$ in LPS-stimulated WT primary microglia, the effects were abolished in Trem2KO microglia (Figures 2B-D). It is noteworthy that the amounts of these inflammatory cytokines were significantly higher in Trem2-KO microglia than WT microglia, which is consistent with our previous reports (Zheng et al., 2016). Taken together, we conclude that DAP12 suppresses the production 

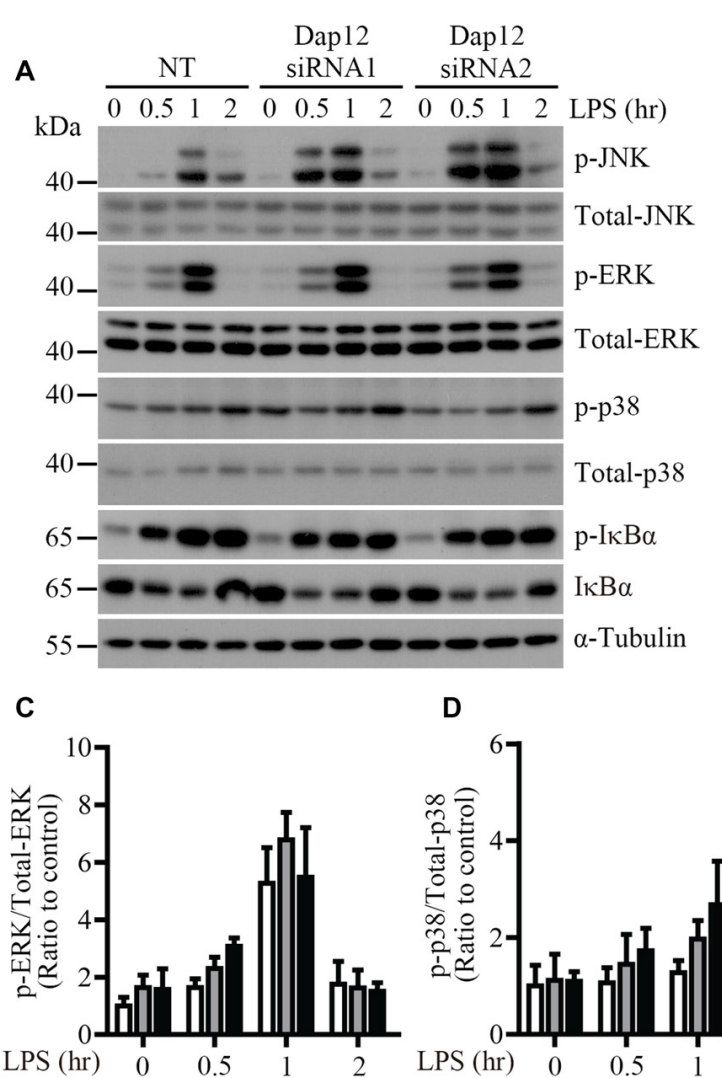

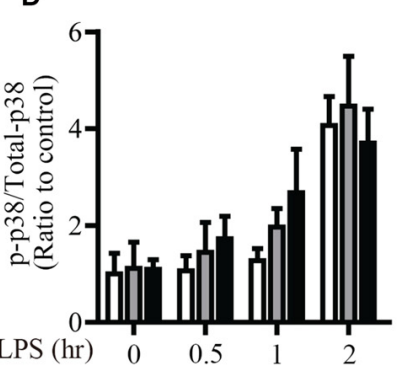

B

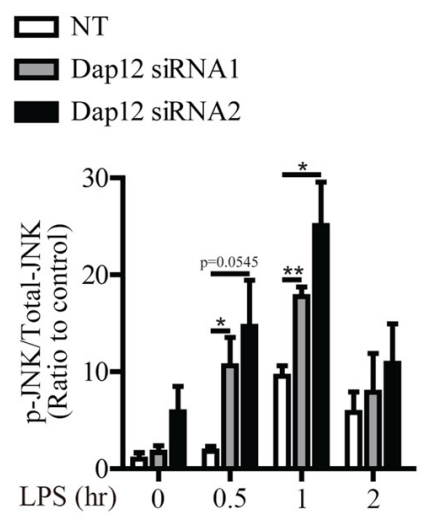

E

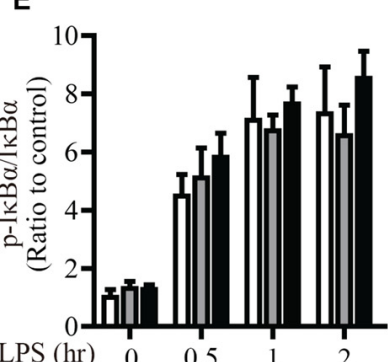

FIGURE 4 | LPS-induced JNK phosphorylation is increased in Dap12-knockdown BV2 cells. (A) BV2 cells were transiently transfected with NT or Dap12-specific siRNAs for $48 \mathrm{~h}$, then stimulated with $500 \mathrm{ng} / \mathrm{mL}$ LPS for the indicated times. (B-E) Bar graphs show the quantification of Western blots as ratios of

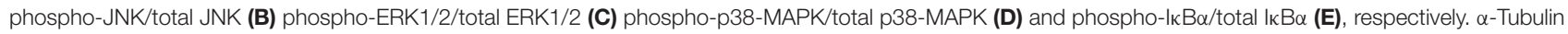
was used as an internal control. The ratio at "O" time point of NT cells served as a control $(n=3$, unpaired Student's $t$-test). Data represent mean \pm SEM. * $p<0.05$; $* * p<0.01$

of pro-inflammatory cytokines in microglia in a manner that depends on TREM2 receptor.

\section{Enhanced JNK Phosphorylation in Trem2- and Dap12-Deficient Microglia}

To identify the signaling pathway(s) that mediates the inflammatory responses induced by LPS in the absence of Trem2/Dap12, we first examined the activation kinetics of the transcription factor NF- $\mathrm{B}$ and the major MAPKs subtypes (ERK1/2, p38-MAPK and JNK) in both WT and Trem2-KO primary microglia. The activation kinetics and magnitude of phosphorylated ERK1/2, p38-MAPK and NF- $\kappa$ B were similar in LPS-stimulated WT and Trem2-KO primary microglia (Figures 3A,C-E). In contrast, the phosphorylation of JNK was more pronounced in primary microglia from Trem2-KO mice compared with WT mice (Figures 3A,B). Similarly, knockdown of Dap12 in microglial BV2 cells significantly increased the phosphorylation of JNK (Figures 4A,B), whereas no effects were observed for phosphorylated ERK1/2, p38-MAPK and ІкB $\alpha$, a key regulator in the NF- $\mathrm{B}$ signaling pathway (Figures $4 \mathrm{C}-\mathrm{E}$ ). We therefore conclude that the TREM2/DAP12 complex regulates the inflammatory responses in microglia by specifically blocking the activation of JNK signaling pathway.

\section{JNK Inhibitor Eliminates the Hypersensitivity of Dap12-Deficient Microglia to LPS}

To further explore the molecular mechanism by which Dap12 down-regulation affects the pro-inflammatory responses induced by LPS, a specific inhibitor (SP600125) was used to block the activation of JNK signaling pathway. Microglial BV2 cells were pre-treated with SP600125 before LPS stimulation. At a dose of 10 or $20 \mu \mathrm{M}$, SP600125 inhibits the phosphorylation of c-Jun which is a downstream target of JNK pathway (Figures 5A,B). The mRNA levels of pro-inflammatory cytokines IL-1 $\beta$ and IL- 6 were increased upon knockdown of Dap12; however, the effect was abolished by pre-treatment with the JNK inhibitor (Figures 5C,D). Taken together, these data indicated that DAP12 negatively regulates LPS-induced inflammatory responses in microglia by modulating the activity of JNK signaling pathway. 
A

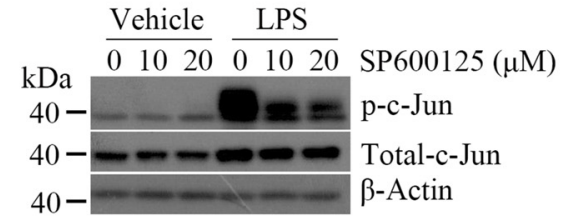

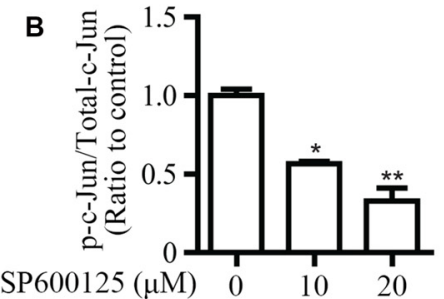

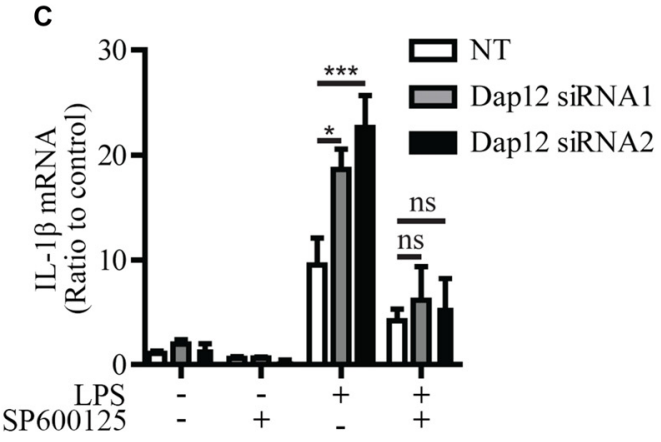

D

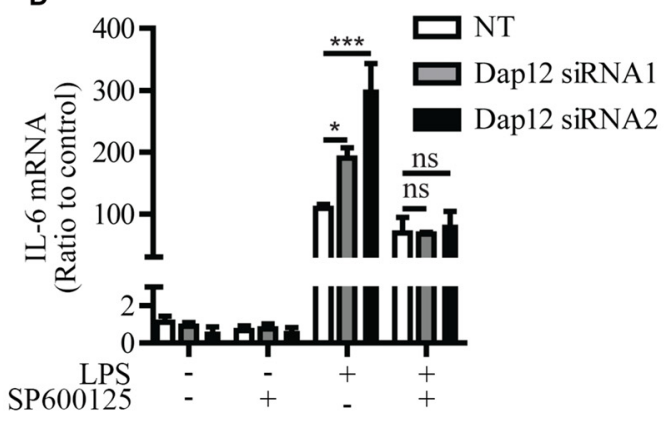

FIGURE 5 | JNK inhibitor reverses the increased pro-inflammatory cytokines in Dap12-knockdown BV2 cells. (A) BV2 cells were pretreated with indicated concentrations of SP600125 for 30 min, and then stimulated with 500 ng/mL LPS or vehicle control for 1 h. Cell lysates were analyzed by Western blotting. (B) Bar graphs show the quantification of Western blots as ratios of phospho-c-Jun/total c-Jun ( $n=3$, one-way ANOVA). (C,D) BV2 cells were transiently transfected with non-targeting siRNA (NT) or Dap 12-specific siRNAs for $48 \mathrm{~h}$, and then stimulated with $500 \mathrm{ng} / \mathrm{mL}$ LPS or vehicle control for $4 \mathrm{~h}$ in the presence or absence of $20 \mu \mathrm{M}$ SP600125 (pretreated for 30 min). RNA was extracted and the relative mRNA levels of IL-1 $\beta$ and IL- 6 shown as bar graph were determined by quantitative RT-PCR $\left(n=3\right.$, two-way ANOVA). $\beta$-actin was used as an internal control. ${ }^{*} p<0.05$; ${ }^{* *} p<0.01$; *** $p<0.001$; ns, not significant.

\section{LPS-Induced Down-Regulation of Trem2 is Rescued by JNK and NF-кB Inhibitors}

We have previously shown that LPS stimulation significantly suppressed Trem 2 expression in primary microglia and mouse brain (Zheng et al., 2016). Consistently, the mRNA levels of Trem2 were significantly down-regulated in LPS stimulated microglial BV2 cells (Figure 6A). However, the expression of Dap12 was unaffected even upon the stimulation with $1 \mu \mathrm{g} / \mathrm{mL}$ LPS (Figure 6B). To further dissect the molecular pathway that modulates Trem 2 expression, we pretreated BV2 cells with various compounds that specifically block individual signaling pathways downstream of LPS, including NF-кB and each of the major MAP kinase subtypes. The mRNA level of Trem 2 was similarly down-regulated by LPS in the presence or absence of p38-MAPK and ERK1/2 inhibitors (Figures 6D,E). However, the LPS down-regulated Trem2 expression was restored by the presence of inhibitors for either JNK or NF- $\mathrm{B}$ (Figures 6C,F). Taken together, our data suggest that both JNK and NF- $\kappa B$ signaling pathways downstream of LPS modulate the expression of Trem 2 in microglia.

\section{DISCUSSION}

In this study, we showed that DAP12 suppresses the production of pro-inflammatory cytokines when microglial cells are exposed to LPS. Importantly, the negative modulation of inflammatory response by DAP12 depends on the presence of TREM2. In view of the underlying molecular mechanism, we revealed that the TREM2/DAP12 axis suppresses the activity of JNK signaling pathway to reduce the inflammatory response in microglia (Figure 7). Intriguingly, LPS down-regulates the expression of Trem 2 via the activation of JNK and NF- $\mathrm{BB}$ signaling pathways (Figure 7), resulting in a vicious cycle that synergistically promotes the inflammatory responses.

As a signaling adaptor protein, DAP12 couples with a variety of cell-surface receptors to modulate the threshold for cellular activation in response to pathogenic stimuli (Turnbull and Colonna, 2007). For instance, the association of DAP12 with TREM1 has been shown to amplify the inflammatory response (Bouchon et al., 2000; Gibot et al., 2004); while the association with TREM2 dampens the production of pro-inflammatory cytokines (Takahashi et al., 2005; Turnbull et al., 2006). Therefore, the roles of DAP12 in different cell types could be varied depending on the presence of specific cell surface receptors. DAP12 is preferentially expressed in microglia within the CNS (Hickman et al., 2013). In our previous work and in current study, we have demonstrated that DAP12 inhibits the production of pro-inflammatory cytokines in LPS-stimulated microglia by using both immortalized cell line and primary cultures (Zhong et al., 2015). We further demonstrated that DAP12 exerts its anti-inflammatory function by coupling with TREM2 which is the highest expressed DAP12-associated receptor in microglia among TREM family members (Zhang et al., 2014). Interestingly, the DAP12 signaling has been shown to amplify inflammation during sepsis (Turnbull et al., 2005). The receptors that are involved remain unknown; TREM1, for instance, might be needed for DAP12 to signal 

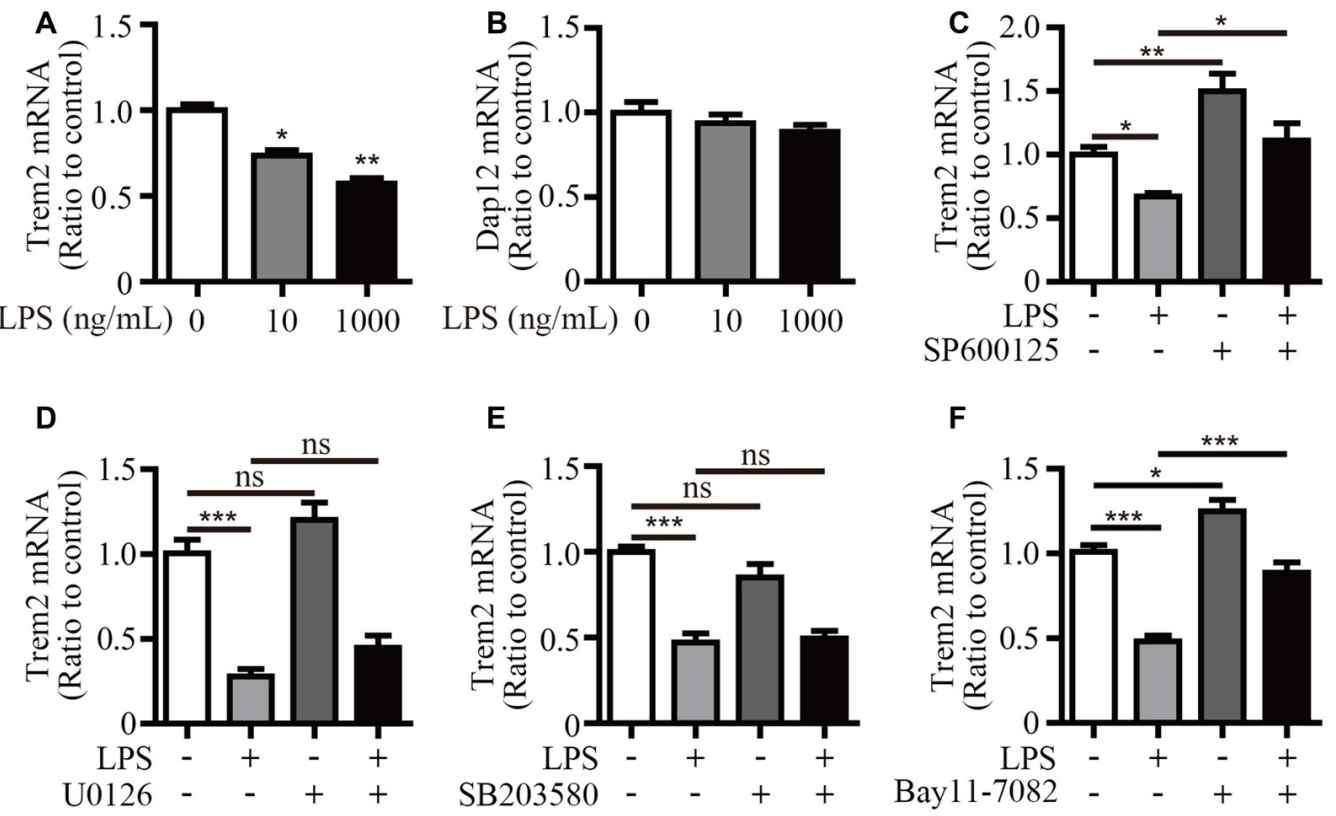

FIGURE 6 | Inhibitors for JNK and NF-кB restore Trem2 expression suppressed by LPS. (A,B) BV2 cells were treated with indicated concentrations of LPS (0, 10 and $1000 \mathrm{ng} / \mathrm{mL}$ ) for $4 \mathrm{~h}$. RNA was extracted and the relative mRNA levels of Trem2 (A) or Dap12 (B) shown as bar graph was determined by quantitative RT-PCR ( $n=3$, one-way ANOVA). (C-F) BV2 cells were pretreated with $10 \mu \mathrm{M}$ SP600125 (C), $5 \mu \mathrm{M} \cup 0126$ (D), $5 \mu \mathrm{M}$ SB203580 (E) or $5 \mu \mathrm{M}$ Bay11-7082 (F) for $30 \mathrm{~min}$, followed by treatment with $500 \mathrm{ng} / \mathrm{mL}$ LPS or vehicle control for $12 \mathrm{~h}$. RNA was extracted and the relative mRNA levels of Trem2 shown as bar graph were determined by quantitative RT-PCR ( $n \geq 3$, one-way ANOVA). $\beta$-actin was used as an internal control. ${ }^{*} p<0.05$; ${ }^{* *} p<0.01$; *** $p<0.001$; ns, not significant.

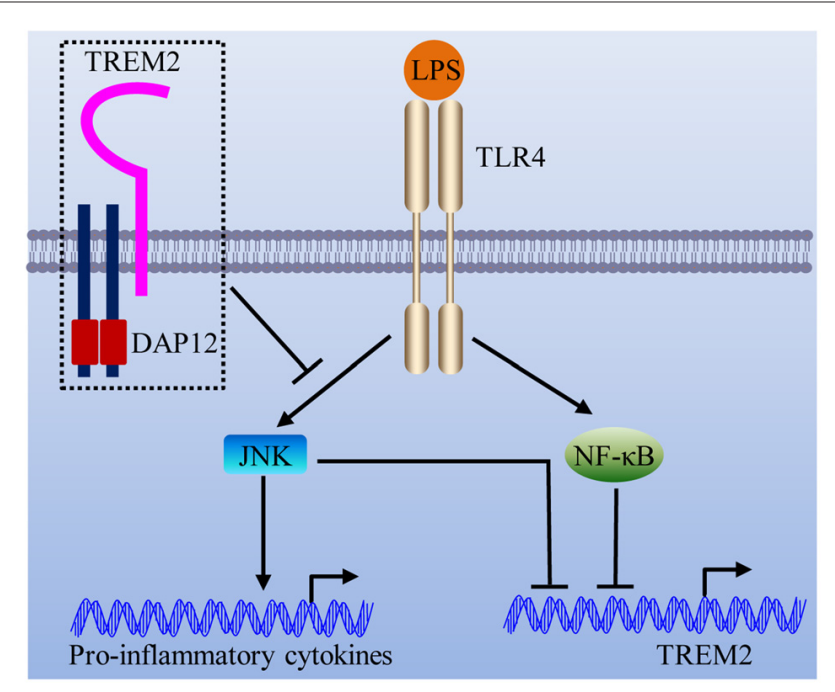

FIGURE 7 | Schematic model of the interplay between TREM2/DAP12 complex and LPS-induced inflammation. The TREM2/DAP12 complex suppresses the activity of JNK signaling pathway to reduce LPS-triggered inflammatory response in microglia. LPS down-regulates the expression of Trem2 via the activation of JNK and NF-kB signaling pathways.

in a pro-inflammatory manner. The activating and inhibitory functions of DAP12 in inflammation are proposed to be modulated by the avidity of the interaction between the DAP12associated receptor and its ligand (Turnbull and Colonna, 2007).
In accordance with our findings in microglia, Dap12-deficient macrophages have been reported to express higher amounts of inflammatory cytokines in response to a variety of pathogenic stimuli (Hamerman et al., 2005). However, the signaling mechanism by which Dap12 regulates cytokine production was distinct between microglia and macrophages. Upon LPS stimulation, ERK1/2 signaling was more pronounced in Dap12deficient macrophages than in WT cells (Hamerman et al., 2005). In contrast, we observed the activation of JNK signaling pathway in Trem2- and Dap12-deficient microglia. It remains uncharacterized how DAP12 regulates the phosphorylation of either ERK1/2 in macrophages or JNK in microglia. Further study is needed to define the precise molecular pathway downstream of DAP12 actions.

We and others have consistently shown that LPS stimulation significantly suppressed microglial Trem2 expression both in vitro and in vivo (Schmid et al., 2002; Zheng et al., 2016). The decrease in Trem 2 expression further augments the production of inflammatory cytokines, leading to detrimental exaggeration of neuroinflammation (Zhong et al., 2015; Zheng et al., 2016). Therefore, understanding the molecular mechanism by which LPS or other pathogenic stimuli regulate Trem 2 expression would provide insights into eliminating the source of inflammation cascade. Our current study showed that applying either JNK or NF- $\mathrm{B}$ inhibitor restored Trem2 expression down-regulated by LPS, implicating a potentially beneficial effect of those inhibitors for treating neurological diseases with an inflammatory component. The precise molecular pathways 
downstream of JNK and NF- $\mathrm{BB}$ require further investigation. It would be intriguing to examine whether the transcription factors activated by JNK and NF- $\mathrm{\kappa B}$ regulate Trem 2 expression via direct binding to its proximal promoter.

Collectively, our studies revealed that DAP12 possesses an anti-inflammatory function in murine microglia that is TREM2dependent. The TREM2/DAP12 axis negatively regulates the activity of JNK signaling pathway downstream of LPS to suppress the inflammatory responses. Our study provides insights into mechanism-based therapy for neuroinflammatory disorders.

\section{AUTHOR CONTRIBUTIONS}

LZ, X-FC and GB: designed research; LZ, Z-LZ, XL, CL, PM, TW, ZQW, ZW and MW: performed experiments; LZ, Z-LZ, XL, CL

\section{REFERENCES}

Atagi, Y., Liu, C. C., Painter, M. M., Chen, X. F., Verbeeck, C., Zheng, H., et al. (2015). Apolipoprotein $\mathrm{E}$ is a ligand for triggering receptor expressed on myeloid cells 2 (TREM2). J. Biol. Chem. 290, 26043-26050. doi: 10.1074/jbc. M115.679043

Bailey, C. C., DeVaux, L. B., and Farzan, M. (2015). The triggering receptor expressed on myeloid cells 2 binds apolipoprotein E. J. Biol. Chem. 290, 26033-26042. doi: 10.1074/jbc.M115.677286

Bouchon, A., Dietrich, J., and Colonna, M. (2000). Cutting edge: inflammatory responses can be triggered by TREM-1, a novel receptor expressed on neutrophils and monocytes. J. Immunol. 164, 4991-4995. doi: 10.4049/jimmunol.164.10.4991

Chung, D. H., Seaman, W. E., and Daws, M. R. (2002). Characterization of TREM3 , an activating receptor on mouse macrophages: definition of a family of single Ig domain receptors on mouse chromosome 17. Eur. J. Immunol. 32, 59-66. doi: 10.1002/1521-4141(200201)32:1<59::AID-IMMU59>3.0.CO;2-U

Daws, M. R., Lanier, L. L., Seaman, W. E., and Ryan, J. C. (2001). Cloning and characterization of a novel mouse myeloid DAP12-associated receptor family. Eur. J. Immunol. 31, 783-791. doi: 10.1002/1521-4141(200103)31:3<783::AIDIMMU783>3.0.CO;2-U

Gibot, S., Kolopp-Sarda, M. N., Béné, M. C., Bollaert, P. E., Lozniewski, A., Mory, F., et al. (2004). A soluble form of the triggering receptor expressed on myeloid cells-1 modulates the inflammatory response in murine sepsis. J. Exp. Med. 200, 1419-1426. doi: 10.1084/jem.200 40708

Guerreiro, R., Wojtas, A., Bras, J., Carrasquillo, M., Rogaeva, E., Majounie, E., et al. (2013). TREM2 variants in Alzheimer's disease. N. Engl. J. Med. 368, 117-127. doi: 10.1056/NEJMoa1211851

Hamerman, J. A., Jarjoura, J. R., Humphrey, M. B., Nakamura, M. C., Seaman, W. E., and Lanier, L. L. (2006). Cutting edge: inhibition of TLR and FCR responses in macrophages by triggering receptor expressed on myeloid cells (TREM)-2 and DAP12. J. Immunol. 177, 2051-2055. doi: 10.4049/jimmunol.177.4.2051

Hamerman, J. A., Tchao, N. K., Lowell, C. A., and Lanier, L. L. (2005). Enhanced Toll-like receptor responses in the absence of signaling adaptor DAP12. Nat. Immunol. 6, 579-586. doi: 10.1038/ni1204

Hickman, S. E., Kingery, N. D., Ohsumi, T. K., Borowsky, M. L., Wang, L. C., Means, T. K., et al. (2013). The microglial sensome revealed by direct RNA sequencing. Nat. Neurosci. 16, 1896-1905. doi: 10.1038/nn.3554

Huang, D., Zimmerman, M. I., Martin, P. K., Nix, A. J., Rosenberry, T. L., and Paravastu, A. K. (2015). Antiparallel $\beta$-sheet structure within the C-terminal region of 42-residue Alzheimer's amyloid- $\beta$ peptides when they form 150-kDa oligomers. J. Mol. Biol. 427, 2319-2328. doi: 10.1016/j.jmb.2015. 04.004

Humphrey, M. B., Lanier, L. L., and Nakamura, M. C. (2005). Role of ITAM-containing adapter proteins and their receptors in the immune and X-FC: analyzed data; X-FC and LZ: wrote the manuscript; HX and GB: reviewed the manuscript. All authors read and approved the final manuscript.

\section{FUNDING}

Research by the authors was supported by grants from the National Natural Science Foundation of China 81370459, 31400914 (to X-FC) and U1505227 (to GB), a grant from the Fundamental Research Funds for the Central Universities (20720150051 to X-FC), a grant from the Guangdong Natural Science Foundation (2016A030306005 to X-FC), NIH grants R01AG035355 and P01NS074969 (to GB), a grant from the Cure Alzheimer's Fund (to GB), a grant from the China Postdoctoral Science Foundation (2016M600503 to LZ).

system and bone. Immunol. Rev. 208, 50-65. doi: 10.1111/j.0105-2896.2005 00325.x

Jay, T. R., Miller, C. M., Cheng, P. J., Graham, L. C., Bemiller, S., Broihier, M. L., et al. (2015). TREM2 deficiency eliminates TREM2+ inflammatory macrophages and ameliorates pathology in Alzheimer's disease mouse models. J. Exp. Med. 212, 287-295. doi: 10.1084/jem.20142322

Jonsson, T., Stefansson, H., Steinberg, S., Jonsdottir, I., Jonsson, P. V., Snaedal, J., et al. (2013). Variant of TREM2 associated with the risk of Alzheimer's disease. N. Engl. J. Med. 368, 107-116. doi: 10.1056/NEJMoa1211103

Lanier, L. L. (2009). DAP10- and DAP12-associated receptors in innate immunity. Immunol. Rev. 227, 150-160. doi: 10.1111/j.1600-065x.2008.00720.x

Lanier, L. L., and Bakker, A. B. (2000). The ITAM-bearing transmembrane adaptor DAP12 in lymphoid and myeloid cell function. Immunol. Today 21, 611-614. doi: 10.1016/s0167-5699(00)01745-x

Lanier, L. L., Corliss, B. C., Wu, J., Leong, C., and Phillips, J. H. (1998). Immunoreceptor DAP12 bearing a tyrosine-based activation motif is involved in activating NK cells. Nature 391, 703-707. doi: 10.1038/35642

Mason, L. H., Willette-Brown, J., Taylor, L. S., and McVicar, D. W. (2006). Regulation of Ly49D/DAP12 signal transduction by Src-family kinases and CD45. J. Immunol. 176, 6615-6623. doi: 10.4049/jimmunol.176.11.6615

Paloneva, J., Kestilá, M., Wu, J., Salminen, A., Böhling, T., Ruotsalainen, V., et al. (2000). Loss-of-function mutations in TYROBP (DAP12) result in a presenile dementia with bone cysts. Nat. Genet. 25, 357-361. doi: 10.1038/77153

Paloneva, J., Manninen, T., Christman, G., Hovanes, K., Mandelin, J., Adolfsson, R., et al. (2002). Mutations in two genes encoding different subunits of a receptor signaling complex result in an identical disease phenotype. Am. J. Hum. Genet. 71, 656-662. doi: 10.1086/342259

Pottier, C., Ravenscroft, T. A., Brown, P. H., Finch, N. A., Baker, M., Parsons, M., et al. (2016). TYROBP genetic variants in early-onset Alzheimer's disease. Neurobiol. Aging 48, 222.e9-222.e15. doi: 10.1016/j.neurobiolaging.2016. 07.028

Schmid, C. D., Sautkulis, L. N., Danielson, P. E., Cooper, J., Hasel, K. W., Hilbush, B. S., et al. (2002). Heterogeneous expression of the triggering receptor expressed on myeloid cells- 2 on adult murine microglia. J. Neurochem. 83, 1309-1320. doi: 10.1046/j.1471-4159.2002.01243.x

Sessa, G., Podini, P., Mariani, M., Meroni, A., Spreafico, R., Sinigaglia, F., et al. (2004). Distribution and signaling of TREM2/DAP12, the receptor system mutated in human polycystic lipomembraneous osteodysplasia with sclerosing leukoencephalopathy dementia. Eur. J. Neurosci. 20, 2617-2628. doi: 10.1111/j. 1460-9568.2004.03729.x

Sirkis, D. W., Bonham, L. W., Aparicio, R. E., Geier, E. G., Ramos, E. M., Wang, Q., et al. (2016). Rare TREM2 variants associated with Alzheimer's disease display reduced cell surface expression. Acta Neuropathol. Commun. 4:98. doi: 10.1186/s40478-016-0367-7

Takahashi, K., Rochford, C. D., and Neumann, H. (2005). Clearance of apoptotic neurons without inflammation by microglial triggering receptor expressed on myeloid cells-2. J. Exp. Med. 201, 647-657. doi: 10.1084/jem.20041611 
Turnbull, I. R., and Colonna, M. (2007). Activating and inhibitory functions of DAP12. Nat. Rev. Immunol. 7, 155-161. doi: 10.1038/nri2014

Turnbull, I. R., Gilfillan, S., Cella, M., Aoshi, T., Miller, M., Piccio, L., et al. (2006). Cutting edge: TREM-2 attenuates macrophage activation. J. Immunol. 177, 3520-3524. doi: 10.4049/jimmunol.177.6.3520

Turnbull, I. R., McDunn, J. E., Takai, T., Townsend, R. R., Cobb, J. P., and Colonna, M. (2005). DAP12 (KARAP) amplifies inflammation and increases mortality from endotoxemia and septic peritonitis. J. Exp. Med. 202, 363-369. doi: $10.1084 /$ jem. 20050986

Wang, Y., Cella, M., Mallinson, K., Ulrich, J. D., Young, K. L., Robinette, M. L., et al. (2015). TREM2 lipid sensing sustains the microglial response in an Alzheimer's disease model. Cell 160, 1061-1071. doi: 10.1016/j.cell.2015. 01.049

Yeh, F. L., Wang, Y., Tom, I., Gonzalez, L. C., and Sheng, M. (2016). TREM2 binds to apolipoproteins, including APOE, and CLU/APOJ and thereby facilitates uptake of amyloid- $\beta$ by microglia. Neuron $91,328-340$. doi: 10.1016/j.neuron. 2016.06.015

Yuan, P., Condello, C., Keene, C. D., Wang, Y., Bird, T. D., Paul, S. M., et al. (2016). TREM2 haplodeficiency in mice and humans impairs the microglia barrier function leading to decreased amyloid compaction and severe axonal dystrophy. Neuron 92, 252-264. doi: 10.1016/j.neuron.2016.09.016

Zhang, Y., Chen, K., Sloan, S. A., Bennett, M. L., Scholze, A. R., O’Keeffe, S., et al. (2014). An RNA-sequencing transcriptome and splicing database of glia, neurons and vascular cells of the cerebral cortex. J. Neurosci. 34, 11929-11947. doi: 10.1523/JNEUROSCI.1860-14.2014

Zheng, H., Liu, C. C., Atagi, Y., Chen, X. F., Jia, L., Yang, L., et al. (2016). Opposing roles of the triggering receptor expressed on myeloid cells 2 and triggering receptor expressed on myeloid cells-like transcript 2 in microglia activation. Neurobiol. Aging 42, 132-141. doi: 10.1016/j.neurobiolaging.2016. 03.004

Zhong, L., Chen, X. F., Wang, T., Wang, Z., Liao, C., Wang, Z., et al. (2017). Soluble TREM2 induces inflammatory responses and enhances microglial survival. J. Exp. Med. 214, 597-607. doi: 10.1084/jem.20160844

Zhong, L., Chen, X. F., Zhang, Z. L., Wang, Z., Shi, X. Z., Xu, K., et al. (2015). DAP12 stabilizes the C-terminal fragment of the triggering receptor expressed on myeloid cells-2 (TREM2) and protects against LPS-induced Pro-inflammatory response. J. Biol. Chem. 290, 15866-15877. doi: 10.1074/jbc. M115.645986

Zhu, W., Zheng, H., Shao, X., Wang, W., Yao, Q., and Li, Z. (2010). Excitotoxicity of $\mathrm{TNF} \alpha$ derived from KA activated microglia on hippocampal neurons in vitro and in vivo. J. Neurochem. 114, 386-396. doi: 10.1111/j.1471-4159. 2010.06763.x

Conflict of Interest Statement: The authors declare that the research was conducted in the absence of any commercial or financial relationships that could be construed as a potential conflict of interest.

Copyright (C) 2017 Zhong, Zhang, Li, Liao, Mou, Wang, Wang, Wang, Wei, Xu, Bu and Chen. This is an open-access article distributed under the terms of the Creative Commons Attribution License (CC BY). The use, distribution or reproduction in other forums is permitted, provided the original author(s) or licensor are credited and that the original publication in this journal is cited, in accordance with accepted academic practice. No use, distribution or reproduction is permitted which does not comply with these terms. 